\title{
Les Frères éducateurs et l'enseignement secondaire public québécois (1920-1970)
}

\section{Paul-André Turcotte}

Volume 50, numéro 1, 1983

Bilan de l'histoire religieuse au Canada

Canadian Catholic History: A survey

URI : https://id.erudit.org/iderudit/1007045ar

DOI : https://doi.org/10.7202/1007045ar

Aller au sommaire du numéro

Éditeur(s)

Les Éditions Historia Ecclesiæ Catholicæ Canadensis Inc.

ISSN

0318-6172 (imprimé)

1927-7067 (numérique)

Découvrir la revue

Citer cet article

Turcotte, P.-A. (1983). Les Frères éducateurs et l'enseignement secondaire public québécois (1920-1970). Sessions d'étude - Société canadienne d'histoire de l'Église catholique, 50(1), 231-253. https://doi.org/10.7202/1007045ar

Tous droits réservés @ Les Éditions Historia Ecclesiæ Catholicæ Canadensis Inc., 1983
Ce document est protégé par la loi sur le droit d'auteur. L'utilisation des services d'Érudit (y compris la reproduction) est assujettie à sa politique d'utilisation que vous pouvez consulter en ligne.

https://apropos.erudit.org/fr/usagers/politique-dutilisation/ 


\section{Les Frères éducateurs et l'enseignement secondaire public québécois (1920-1970)}

L'historiographie de l'enseignement au Québec s'attarde généralement à décrire l'emprise de l'Église par le Comité catholique et les collèges classiques avant de passer à la réforme scolaire des années soixante annonçant une ère nouvelle. D'autres études scrutent le système d'enseignement et ses transformations structurelles ou pédagogiques ${ }^{1}$. Les sociologues, de leur côté, tentent de cerner les rapports de l'opération éducative à la société globale ou les conflits entre les divers agents concernés par l'éducation ${ }^{2}$. Les uns et les autres ont en commun de laisser dans l'ombre l'enseignement secondaire public masculin et ses initiateurs, les frères enseignants. À peine se çontente-t-on de signaler l'existence, avant les années soixante, de cet enseignement. Par voie de conséquence, les rares recherches publiées

\footnotetext{
${ }^{1}$ L'auteur le plus connu et le plus prolifique sur l'histoire de l'enseignement québécois est certes Louis-Philippe Audet. Parmi ses nombreux ouvrages, signalons son Histoire de l'enseignement au Québec 1608-1971, dont le second tome concerne la période contemporaine: Histoire de l'enseignement au Québec 1840-1971, Montréal et Toronto, Holt, Rinehart et Winston, 1971, 496 p. Le lecteur y trouvera une bibliographie abondante.

${ }^{2}$ Parmi les études sociologiques d'ensemble, nous retenons deux ouvrages collectifs: Pierre W. Bélanger et Guy Rocher (éds.), École et société au Québec, Montréal, Hurtubise HMH, 1975 [c1970]; Renée Cloutier, Jean Moisset et Jean Ouellet (éds.), Analyse sociale de l'éducation, Montréal, Éditions du Boréal Express, 1983, 346 p. Ces deux ouvrages comprennent des articles, inédits dans le second cas, portant sur différents aspects sociologiques de l'éducation au Québec, et en complément une bibliographie sélective.
} 
sur le sujet, sauf exceptions ${ }^{3}$, sont des publications internes des congrégations concernées, et encore sont-elles fragmentaires.

Dans cet article, notre intention est de rendre compte de l'action des frères enseignants dans le système d'enseignement québécois, depuis les années vingt jusqu'aux années soixante-dix, tel que le révèle la Fédération des Frères Éducateurs (FFE). Celle-ci consiste en la réunion périodique des supérieurs provinciaux des congrégations enseignantes masculines, laïques ou mixtes ${ }^{4}$, localisées principalement dans le périmètre québécois. Au cours de ces réunions, les supérieurs, avec ou sans la présence d'autres religieux intéressés, abordent des questions d'intérêt commun et prennent des décisions relatives à leur intervention dans le système d'enseignement québécois, à la rémunération de leur tâche et à leurs rapports avec d'autres agents sociaux que sont l'État, l'Église, des associations éducatives nationales, les congrégations féminines ou cléricales, les enseignants laïcs.

\footnotetext{
${ }^{3}$ Des ouvrages accordent une attention rapide à notre sujet. À titre d'exemples: Bernard Lefebvre, L'école sous la mitre, Montréal, Éditions Paulines, 1980, 273 p; Arthur Tremblay, Les collèges et les écoles publiques: Conflit ou coordination? Québec, Presses universitaires Laval, 1954, 140 p. René Fandrich fait l'histoire des origines de l'enseignement secondaire public dans L'école primaire supérieure, Montréal, Albert Lévesque, c1934, 183 p. Sur l'évolution de cette question, nous renvoyons à l'excellente thèse de Georges Croteau, Les frères éducateurs au service de la promotion des étudiants dans l'enseignement public au Québec, 1920 à 1960 (Ottawa, Faculté d'Éducation de l'Université d'Ottawa. 1971, 250 p. [polytypé]). Maurice Lebel se fait l'écho de cette thèse dans son Évolution de l'enseignement québécois pendant l'entre-deux guerres (1920-1940), Montréal, Éditions Paulines, 1982, 37 p. Quant aux congrégations religieuses concernées, nous relevons les titres de sociologie historique suivants: Bernard Denault et Benoît Lévesque, Éléments pour une sociologie des communautés religieuses au Québec, Montréal, Les Presses de l'Université de Montréal/Sherbrooke, Université de Sherbrooke, 1975, 220 p.; Gabriel Dussault Charisme et économie. Les cinq premières communautés masculines établies au Québec sous le régime anglais (1837-1870), Québec, Université Laval, Département de Sociologie, 1981, 149 p.; Paul-André Turcotte, L'éclatement d'un monde. Les Clercs de SaintViateur et la révolution tranquille, Montréal, Bellarmin, 1981, 366 p.

${ }^{4}$ D'un point de vue structurel, l'Église catholique est composée de clercs et de laïcs (structure institutionnelle ou sacramentelle), ceux-ci des non-religieux ou séculiers en opposition aux religieux (structure charismatique). Les frères enseignants sont des religieux-laïcs, car ils appartiennent à l'état de vie religieux dans l'ordre charismatique et ils sont assimilés au laïcat en tant que non-clercs dans l'ordre institutionnel. De plus, ils sont regroupés dans une congrégation que l'on dira laïque, ce terme devant être compris en rapport à une congrégation cléricale qui réunit des prêtres auxquels peuvent s'ajouter des frères subalternes, dits convers ou coadjuteurs. Une congrégation mixte comprend sur un même pied, au moins en principe, des prêtres ou religieuxclercs et des frères ou religieux-laïcs, avec ou sans frère convers; c'est le cas, au Canada et parmi les congrégations enseignantes, des Clercs de Saint-Viateur, des Marianistes, et jusqu'en 1948, de la Congrégation de Sainte-Croix. La catégorie des congrégations mixtes n'est pas reconnue par le droit universel de l'Église.
} 
Nous nous limiterons à retracer la genèse et l'évolution de la Fédération ${ }^{5}$, à montrer le rapport entre son institutionnalisation, c'est-àdire sa coordination impérative interne, le degré d'autonomie ou de subordination institutionnelle des congrégations et leur capacité d'institution, à savoir leur participation à la formulation et à la réalisation de projets éducatifs innovateurs. Ce faisant, nous dégagerons certains aspects de la pratique des congrégations enseignantes masculines québécoises en regard de l'enseignement secondaire public pendant un demisiècle.

\section{UNE INTERVENTION CRÉATRICE LIMITÉE PAR LA SUBORDINATION INSTITUTIONNELLE (1919-1945).}

Le 10 juin 1901, le Frère Ulysse, provincial des Frères de l'Instruction chrétienne (FIC), écrivit au Père Charles Ducharme, son homologue des Clercs de Saint-Viateur canadiens (CSV), qu'il incombait aux congrégations de se concerter afin de mieux définir leurs conditions d'embauche auprès des commissions scolaires et de la sorte échapper aux manoeuvres dont elles étaient l'objet. L'année suivante, les FIC durent abandonner le Collège de Saint-Cuthbert, et le Frère Ulysse revint à la charge pour offrir l'établissement aux CSV déjà à l'oeuvre dans la région. Il faut attendre l'année 1918 pour que les provinciaux des frères enseignants reprennent l'idée d'un rapprochement intercongréganiste grâce à la formation d'un comité permanent à Montréal. À l'uniformisation des rapports avec les commissions scolaires s'ajoutaient désormais d'autres questions: les salaires, les manuels, les examens et programmes, la propagande sur le travail des frères et l'accord, au lieu de la divergence, sur les problèmes et l'action dans l'enseignement ${ }^{6}$.

\footnotetext{
${ }^{5}$ Le présent article s'inscrit dans le cadre d'une recherche post-doctorale sur les rapports entre fonction et utopie dans le catholicisme québécois, notamment dans le champ de l'éducation, recherche menée grâce à une subvention du Conseil de Recherches en Sciences humaines du Canada. Nos propos s'appuient sur le dépouillement des archives volumineuses de la FFE et des entrevues avec des acteurs encore vivants. En toile de fond peut-on apercevoir nos recherches antérieures sur les congrégations enseignantes québécoises.

${ }^{6}$ Archives de la Fédération des Frères éducateurs (désormais AFFE), Cyrille Côté, FEC, "25, 30, 60 ans d'étroite collaboration", Frères éducateurs, 21 (janvier-février 1970), p. 34; AFFE, Projet d'un comité permanent des communautés religieuses enseignantes, 1918, $1 \mathrm{p}$.
} 
Les provinciaux exprimèrent, lors des rencontres préparatoires de 1918 et de 1919, le besoin de resserrer les rangs. Il s'imposait, d'après eux, de défendre les intérêts des congrégations et de maintenir leurs droits par l'étude des questions d'utilité commune. Divers événements les poussaient en ce sens. Les laïcs réussissaient à faire valoir leurs positions auprès des commissions scolaires, voire du grand public, en faisant publier dans les journaux les résolutions de leurs cercles professionnels récemment constitués. De plus, l'état matériel des résidences scolaires n'avait cessé de se détériorer, et les commissions scolaires de l'île de Montréal avaient commencé de se regrouper, en 1916-1917, en secteurs géographiques administrés par un directeursecrétaire aux pouvoirs décisionnels menaçant ceux des congrégations, notamment dans le District Nord ?

Les supérieurs provinciaux des congrégations à l'oeuvre dans ce district scolaire se réunirent à la Maison provinciale des CSV le 18 octobre 1919. Ces congrégations étaient les Clercs de Saint-Viateur, la Congrégation de Sainte-Croix (CSC), les Frères Maristes (FMS), les Frères de Saint-Gabriel (FSG) et les Frères de l'Instruction chrétienne. À cette séance et par la suite, les provinciaux affirmèrent l'unanimité de leur position devant une certaine centralisation des organes de décision scolaires et leur intention de garder le contrôle traditionnel de l'école, surtout en ce qui regarde l'affectation professionnelle de leurs sujets, les objectifs éducatifs et la pratique de la règle religieuse ${ }^{8}$.

Une telle visée requérait la participation des Frères des Écoles chrétiennes (FEC) particulièrement influents auprès des autorités scolaires. Au printemps de 1920, des démarches furent entreprises pour que les FEC intègrent le comité. Ils le firent par étapes et non sans réticences ${ }^{9}$. D'autres congrégations se grefferent au rameau initial: les Frères du Sacré-Coeur (FSC) et les Frères de la Charité (FC), de sorte qu'en 1940 la Fédération comptait huit congrégations réparties en onze provinces religieuses. Soulignons la présence sporadique des Presentation Brothers. La Fédération avait dépassé rapidement les frontières du District Nord de Montréal pour s'étendre à la grandeur du Québec, gardant ses bureaux dans la métropole canadienne.

\footnotetext{
${ }^{7}$ Marcel de Grandpré, CSV, Le Père Alphonse de Grandpré csv, Joliette, Maison provinciale des Clercs de Saint-Viateur, 1954, pp. 51-2.

${ }^{8}$ AFFE, Lettre du Père J.-A. Charlebois, CSV, aux supérieurs provinciaux du District Nord, 13 octobre 1919; AFFE, Rapport de la réunion, 24 avril 1920, p. 1; 22 mai 1920, p. 1.

${ }^{9}$ AFFE, Rapport de la réunion, 24 avril 1920, p. 1; 22 mai 1920, p. 1.
} 
Toujours en vue de renforcer le poids social, les provinciaux invitèrent les congrégations féminines à se joindre à eux. Elles n'acquiescèrent pas à cette invitation, souhaitant toutefois être informées des démarches des frères auprès des commissions scolaires ${ }^{10}$. Dans le même sens, la présidence fut confiée d'office au provincial des Clercs de SaintViateur résidant à Outremont. Dans une société cléricalisée, les frères enseignants avaient comme porte-parole un prêtre officier majeur d'une congrégation composée majoritairement de non-clercs et reconnue socialement en raison de son ancienneté, de son nombre, de sa canadianisation et de ses apports à la collectivité.

Forts d'une intention commune et d'un certain poids social, les supérieurs s'imposèrent aux instances civiles et religieuses. Ils affirmèrent d'abord leur intention ferme de garder le contrôle de leurs sujets à l'emploi des commissions scolaires. Pour ce, ils défendirent l'autonomie des écoles et des directeurs religieux par rapport à l'administration scolaire centrale. La gamme des doléances et mesures s'étendit de l'uniformisation des bulletins ou du prix des fournitures scolaires, si ce n'est l'achat de la craie, à la définition de la tâche du directeur, de son adjoint ou des enseignants, laïques et religieux. Il importait au premier chef que le supérieur provincial et son subalterne, le directeur, puissent voir à la marche de l'école dans sa totalité. D'où la recommandation faite au directeur-secrétaire, dans l'introduction de changements, de consulter les provinciaux des congrégations avec qui les commissions scolaires concluaient des contrats.

Il fallait aussi veiller à l'observance de la règle et au bien-être physique des religieux. Ainsi les supérieurs s'en prirent-ils à l'obligation de surveiller l'étude des élèves dans la salle de classe après une journée déjà surchargée. De même, les conférences obligatoires pour les enseignants avaient lieu à des heures inconciliables avec l'horaire des exercices de piété ${ }^{11}$. Ces questions ponctuelles engagèrent des négociations parfois serrées où les supérieurs s'imposèrent, à moins de consentir à des concessions mineures. Les réclamations, nombreuses et pressantes

\footnotetext{
${ }^{10}$ Soeur Marie de Sainte-Croix, CSC, écrivit au Père J.-A. Charlebois, CSV, le 6 décembre 1919: «Les Congrégations d'hommes ont plus que nous l'autorité qui permet d'élever la voix, cependant nous avons cru devoir aussi porter devant les directeurs quelques demandes justifiées, mais il est bon de vous sentir les premiers et de nous appuyer sur vous" (AFFE).

${ }^{11}$ AFFE, Procès-verbal, 18 octobre 1919, pp. 1-2; 15 novembre p. 1; 8 août 1923, 1 p.; 8 janvier 1928, 2 p; AFFE, Lettre du P. J.-A. Charlebois, CSV, aux présidents des Commissions scolaires de district, 21 février 1920.
} 
dans les années vingt, devinrent espacées dans la décennie suivante. Peut-être était-ce dû à la représentation des frères auprès du Comité catholique et de la Commission scolaire de Montréal, ce qui permettait une intervention directe au sein même des organes de décision.

La défense de l'autonomie de l'école avait comme finalité la régulation de l'enseignement, mais un enseignement dont chaque congrégation prétendait à une spécificité. Dans cette ligne, l'uniformisation des manuels scolaires ne recueillit pas l'assentiment du comité des provincieux, malgré les pressions des instances scolaires ${ }^{12}$. De même, les congrégations ne renoncèrent pas à des façons de faire particulières, souvent héritées des origines européennes, même si elles étaient sévè̀rement critiquées dans l'entourage. En outre, l'expansion géographique, le recrutement et l'affirmation d'une singularité éducative suscitaient une concurrence inconciliable avec la mise en commun des ressources matérielles et humaines. Les congrégations s'entendirent toutefois pour ne pas faire le jeu des commissaires profitant de cette concurrence pour déloger une communauté. Elles travaillèrent également à deux projets majeurs: l'implantation du cours primaire supérieur et l'établissement des scolasticats-écoles normales.

En 1931, l'Assemblée législative du Québec adopta une loi à l'effet que le lieutenant-gouverneur en conseil, sur la recommandation du Comité catholique du Conseil de l'Instruction publique, pouvait reconnaître les scolasticats de frères enseignants comme écoles normales, à la condition que ces scolasticats fussent établis et maintenus aux frais des congrégations ${ }^{13}$. C'était l'aboutissement de négociations qui avaient débuté en 1929 grâce à l'appui du cardinal R.-M. Rouleau de Québec et de l'archevêque-coadjuteur de Montréal, $\mathbf{M}^{\text {gr }}$ Gauthier. Les directions congréganistes tenaient à ce que leurs sujets aient des diplômes officiels, car une partie de la population, et particulièrement les enseignants laïques, considéraient comme un privilège désuet la capacité pour les religieux d'enseigner sans diplôme officiel. D'ailleurs, des supérieurs pensaient qu'une disposition de la loi de l'instruction publique abolirait ce privilège dans un avenir prochain ${ }^{14}$.

${ }^{12}$ AFFE, Procès-verbal, 18 octobre 1919, p. 2; Rapport du comité de coordination, 25 avril 1942, p. 2; 21 février 1943; 2 p; Cyrille Côté, FEC, «25, 30, 60 ans...», p. 35.

${ }^{13}$ AFFE, Bill modifiant la loi de l'Instruction publique, $4^{\mathrm{e}}$ session, $17^{\mathrm{e}}$ législature, 21 Georges V, 1930-1931, art. 478a.

${ }^{14}$ AFFE, Procès-verbal, 17 sept. 1930, 1 p; AFFE, Lettre des supérieurs provinciaux au Cardinal Rouleau, 28 décembre 1928 et 25 janvier 1929; J.-A. Larivière, CSV, Marius Soffray clerc de Saint-Viateur, Joliette, Les Clercs de Saint-Viateur, 1975, p. 48. 
L'obtention d'une compétence officiellement reconnue fut assurée par l'autorisation que les frères normaliens passent les mêmes examens que les laïcs. Mais cette disposition ne devrait en rien compromettre la nomination des directeurs ou des professeurs par les supérieurs majeurs. On refusa donc les subventions gouvernementales par crainte d'un contrôle des affaires internes. En outre, la préparation du futur maître religieux avait allié, dans les scolasticats remontant au moins à la première guerre mondiale, l'acquisition de connaissances et l'entrâ̂nement progressif à la vocation (Beruf) d'enseignant. Aussi redoutait-on une mainmise de l'État négligeant cette complémentarité jugée primordiale, car c'est par elle que s'effectuait la socialisation des recrues à la spécificité congrégationnelle ${ }^{15}$.

Un autre facteur joua dans l'établissement d'écoles normales congréganistes: le besoin de maîtres aptes à l'enseignement secondaire public en pleine expansion. En effet, les Clercs de Saint-Viateur avaient mis sur pied le cours primaire supérieur à l'École Saint-Louis du MileEnd en septembre 1921. Une décennie de pressions auprès des commissaires de Montréal avait précédé la naissance d'un enseignement secondaire public autre que le seul enseignement commercial. Il importait de donner aux enfants des classes dites populaires la possibilité de faire des études secondaires menant à l'université et préparant spécialement au commerce, à l'industrie, aux sciences et à l'agriculture. Le jeune Canadien devait avoir autant d'avenir que le jeune Canadien des High Schools ${ }^{16}$.

Cet objectif fut atteint par le premier cours de Saint-Louis, dont plusieurs finissants furent admis à l'université et y décrochèrent des grades ${ }^{17}$. Ceux qui suivirent n'eurent pas cette possibilité du fait qu'en 1929, avec l'approbation officielle du programme, l'enseignement des langues anciennes fut aboli et celui des sciences ou des mathématiques tronqué. Par voie de conséquence, l'accès aux écoles supérieures et aux facultés fut limité aux domaines du commerce, de la polytechnique et

\footnotetext{
${ }^{15}$ Cf. note précédente.

${ }^{16}$ Georges Croteau, Les frères éducateurs, pp. 27-77.

${ }^{17}$ Sept des premiers élevves du cours primaire supérieur de Saint-Louis furent admis en rhétorique au Collège de Rigaud et se classèrent parmi les premiers aux examens de baccalauréat de l'Université de Montréal; deux autres réussirent aux Hautes Études commerciales; six finissants de Saint-Louis, admis en Polytechnique, y figurèrent brillamment. D'autres succès peuvent être relevés aux Beaux-Arts ou à l'Université d'Ottawa (ibid., p. 97).
} 
des sciences ${ }^{18}$. Un sort similaire échut au cours secondaire sans grec et au cours secondaire moderne, les deux créés à Québec par les FEC, respectivement en 1925 et en $1938{ }^{19}$.

Le cours primaire supérieur ne continua pas moins de s'étendre. Le nombre des écoles offrant ce cours, partiellement ou en totalité, passa de 172 en 1929 à 274 en 1940 . À cette date, $30 \%$ des frères enseignants s'adonnaient à l'enseignement secondaire rejoignant 4000 élèves ${ }^{20}$. Les congrégations enseignantes de Québec et de Montréal revinrent à la charge en 1939-1940 pour que le Département de l'Instruction publique fasse du cours primaire supérieur un cours équivalent à celui des écoles secondaires anglo-québécoises. C'était la voie désignée, avançait-on, pour sortir les canadiens français de leur infériorité intellectuelle et économique. Un mémoire particulièrement élaboré fut celui présenté en 1942 par la Commission pédagogique des Frères des Écoles chrétiennes de Québec. Le Comité catholique ne donna pas suite à la plupart des recommandations, dont certaines n'allaient être appliquées que dans les décennies subséquentes. Parmi ces mesures, mentionnons la centralisation et la régionalisation des écoles, le transport des écoliers, la scolarité obligatoire jusqu'à l'âge de quatorze ans, l'enseignement du latin dans le secteur public, la création d'une université d'État ${ }^{21}$.

Il ressort de cette brève description que les frères éducateurs ont su affirmer une certaine autonomie d'intervention dans l'enseignement québécois masculin grâce à la concertation continue, quoique grandement informelle, entre les supérieurs, et ce en confrontation aux autorités scolaires centralisatrices. En raison même de cette autonomie et de l'appui social régional, civil et presbytéral, les CSV, entraînant à leur suite d'autres congrégations, et les FEC dans leurs écoles, ont formulé des projets de réforme scolaire mettant en cause le monopole des humanités classiques dans l'accès au degré supérieur. Mais la pratique resta en deçà des projets souhaités vu les aléas de la concurrence et la subordination des congrégations masculines à l'égard de l'Église officielle contrôlant, de concert avec la bourgeoisie des professions libérales,

\footnotetext{
${ }^{18}$ Ibid., pp. 94-101.

${ }^{19}$ L'Université Laval ne reconnut pas le diplôme de chacun de ces deux cours comme l'équivalent du baccalauréat ès arts. Le titulaire de l'un ou l'autre des diplômes pouvait entrer à l'École supérieure de Commerce, à l'École d'Arpentage et de Génie Forestier ou à la Faculté des Sciences (Maurice Lebel, Évolution de l'enseignement québécois, p. 33 , note 56 ).
}

${ }^{20}$ Ibid., p. 14.

${ }^{21}$ Georges Croteau, Les frères éducateurs, pp. 107-28. 
les pouvoirs de décision dans le domaine de l'éducation. Les congrégations ne se taillèrent pas moins une place de premier plan dans l'enseignement secondaire public.

\section{UNE CONSOLIDATION DU CHAMP D'ACTION FRAGILE EN RAISON DE LA DÉPENDANCE INSTITUTIONNELLE ACCRUE (1945-1960)}

Un secrétariat permanent du comité des provinciaux fut mis sur pied le 25 octobre 1945. Par la même occasion, le comité postula une charte civile à titre de corporation à fin pédagogique appelée la Fédération des Frères Enseignants du Québec. De la sorte les huit congrégations membres se donnaient un moyen institutionnel pour défendre leurs intérêts en ce qui concerne la pédagogie, les manuels scolaires, les salaires, les octrois gouvernementaux, les relations avec l'Alliance catholique des Instituteurs (laïques), et autres questions ${ }^{22}$. Le 5 juin 1959, l'appellation de l'organisme devint la Fédération des Frères Éducateurs du Canada, comprenant désormais 10 congrégations ${ }^{23}$ réparties en 23 provinces totalisant, au 30 décembre de la même année, 6600 religieux, dont 6343 au Québec ${ }^{24}$. La Fédération continua donc de s'étendre et de couvrir ainsi un champ territorial de plus en plus grand.

Les tâches confiées au secrétariat exécutif, composé d'un chef de bureau et de deux adjoints, étaient celles que l'on retrouve habituellement dans ce genre d'organisme: préparation, convocation et procèsverbal des réunions, tenue des archives, correspondance, tractations avec les autorités scolaires, exécution modulée des décisions des pro-

\footnotetext{
${ }^{22}$ AFFE, Procès-verbal, 19 avril 1945, p. 1; AFFE, Acte de la fondation du secrétariat général des frères enseignants du Québec, 25 octobre 1945, 1 p. La signature du document n'aura lieu que le 7 décembre suivant.

${ }^{23}$ Les nouvelles congrégations membres furent les frères de Notre-Dame de la Miséricorde et les Marianistes. En outre, les Presentation Brothers avaient assisté à quelques réunions depuis les années vingt. Fait significatif, les FEC de langue anglaise ne sont pas habituellement comptés parmi les religieux des congrégations membres; il en va autrement chez les CSV incluant même les prêtres. S'il n'y eut pas d'exclusion formelle des anglophones, il semble bien que ceux-ci ne furent pas intéressés à discuter de problèmes propres aux francophones très grandement majoritaires. D'ailleurs, les anglophones catholiques purent jouir des privilèges des High Schools protestants dès la fin des années vingt.

${ }^{24}$ AFFE, Procès-verbal, 5 juin 1959, p. 2; AFFE, Renseignements divers, 30 décembre 1959, p. 1.
} 
vinciaux, communications entre ces derniers et information documentée sur tout sujet digne d'intérêt, particulièrement sur des questions pédagogiques ou sociales. L'acte de fondation précisait que les supérieurs provinciaux devaient se rencontrer, outre les réunions spéciales, au moins cinq fois l'an, soit le deuxième samedi des mois d'octobre, de décembre, de février, d'avril et de juin ${ }^{25}$. Ces règles allaient rester les mêmes jusqu'en 1962. Elles consignaient en de courts textes une pratique d'un quart de siècle, donnant une détermination à ce qui avait été jusque-là grandement informel.

Dès 1963, les supérieurs provinciaux autorisèrent les directeurs des juvénats et les recruteurs des quatorze provinces religieuses représentées à se rencontrer périodiquement et à discuter de points relatifs au recrutement. Ce fut le tour ensuite des procureurs provinciaux, des directeurs des scolasticats-écoles normales, des directeurs des études. En 1954 fut mis sur pied la Commission des Frères des Écoles secondaires avec comme objectif de travailler à la coordination du degré secondaire, et des sous-commissions tâchèrent, de 1956 à 1959, de voir à l'application des nouveaux programmes du Département de l'Instruction publique. Des représentants des enseignants laïques, de l'Action catholique ou d'autres organismes, des supérieurs généraux ou visiteurs, des spécialistes congréganistes en telle matière, furent invités à échanger avec les provinciaux. Le 18 novembre 1959, la Fédération fut réaménagée en sous-comités d'étude portant respectivement sur la vie religieuse, les vocations, l'apostolat, la vie professionnelle et sociale, les finances et la publicité ${ }^{26}$. Les questions à l'étude étaient devenues complexes et requéraient, de ce fait, un éclairage plus grand pour permettre aux supérieurs de prendre des décisions adéquates.

Le réaménagement institutionnel du comité des provinciaux avait été engagé dans le but de procurer des assises plus fermes aux frères dans le système d'enseignement, notamment dans le secondaire. Il fallait aussi trouver une solution aux problèmes financiers consécutifs à la crise économique. De plus, les congrégations étaient obligées d'accorder leur action à une société en ébulition: la seconde industrialisation forçait à des ruptures d'avec la tradition, et des agents sociaux prônaient des

\footnotetext{
${ }^{25} \mathrm{AFFE}$, Acte de fondation du secrétariat général des frères enseignants du Québec, 25 octobre $1945,1 \mathrm{p}$.

${ }^{26}$ AFFE, Lettre du F. M.-Cyrille, FEC, aux frères directeurs des juvénats et aux recruteurs des divers instituts, 13 février 1946 et 11 juin 1946; AFFE, Procès-verbal, 8 juin 1946, p. 3; AFFE, Commission des Frères des Écoles secondaires. Historique, s.d., 1 p.; AFFE, Sujet d'étude pour les divers comités, 18 novembre 1959, 1 p.
} 
changements décisifs dans la société, notamment dans le domaine de l'éducation. Ici, les frères entendaient emboîter le pas tout en consolidant leur position dans les cadres établis. D'ailleurs, l'existence de ces derniers, qu'ils fussent ecclésiastiques ou civils, étaient reconnue par la majorité des contestataires sociaux des années quarante.

Le mouvement de la société québécoise allait avoir des incidences sur la politique des congrégations enseignantes. L'explosion démographique de l'après-guerre entraîna une hausse substantielle de la clientèle scolaire catholique, soit $88 \%$ de 1945 à $1960{ }^{27}$. Le nombre des enseignants s'accrut parallèlement, mais à l'avantage des laïcs dont l'augmentation, de 1956 à 1960, fut de 11507 , comparativement à une diminution de 268 chez les religieux ${ }^{28}$. Ceux-ci continuèrent d'assumer la direction d'un nombre toujours plus élevé d'écoles publiques, même s'ils devaient affronter la concurrence des laïcs désireux de détenir davantage de postes clé dans l'enseignement. Devant cette intention et le danger de minorisation définitive, les frères entreprirent la croisade des vocations, rencontrant sur leur route des forteresses cléricales bien gardées. Des conflits épiques en rapport à ce point éclatèrent au cours des années cinquante: les religieux-laïcs revendiquèrent l'accès à un champ de recrutement plus étendu, ce qui leur fut refusé. Pendant ce temps des évêques et des prêtres s'inquiétaient de la concentration des frères dans l'enseignement secondaire, car elle pouvait tarir la source de recrutement sacerdotal qu'étaient les écoles primaires congéganistes ${ }^{29}$.

En regard de l'enseignement secondaire, les congrégations mirent l'accent sur la formation intellectuelle et professionnelle de leurs membres. Cours du soir, de fin de semaine ou d'été ne cessèrent de prendre de l'importance. Peu à peu se créa un hiatus entre le savoir profane et les connaissances relatives à la religion, ce qui entraîna des défections, tout au moins des malaises profonds chez un grand nombre de religieux. En vue de remédier à cet état de choses et d'assurer une

${ }^{27}$ Les élèves des écoles publiques catholiques passèrent de 493000 en 1945 à 926000 en 1960 , soit une augmentation de $88 \%$, alors que l'accroissement de la population du Québec fut de $40 \%$ pendant la même période (Pierre Angers, SJ, Réflexions sur l'enseignement, Montréal, Bellarmin, 1963, p. 77).

${ }^{28}$ Gérard Dion, "La sécularisation de la société québécoise», dans Collectif, Les valeurs chrétiennes et l'éducation, Québec, Les Presses de l'Université Laval, 1967, p. 64.

${ }^{29}$ Ces questions furent l'objet des délibérations de la Fédération durant les années cinquante sans interruption, à l'égal des problèmes financiers. Des dossiers bien documentés, des colloques et des allocutions publiques portèrent, au-delà de la Fédération, le débat sur des thèmes connexes. 
connaissance plus étendue de la doctrine catholique dans la formation des maîtres d'enseignement religieux, un Institut supérieur de Sciences religieuses ouvrit ses portes à l'Université de Montréal en septembre 1954, suivi par l'école universitaire Jesus Pastor d'Ottawa en $1960{ }^{30}$. Une collaboration plus poussée dans la socialisation des recrues ou la préparation des formateurs n'alla pas toutefois jusqu'à la création, en 1954, d'un scolasticat intercongréganiste, vu l'abstention des Frères du Sacré-Coeur ${ }^{31}$.

La collaboration se remarqua davantage dans la participation à des congrès nationaux et à la journée des fondateurs, autant d'occasions de faire valoir l'action des frères enseignants dans la société et ainsi de revendiquer un prestige social positif. La reconnaissance sociale positive était requise vu la nécessité d'accroître le recrutement, d'affronter les contestataires sociaux et les entraves dans l'enseignement public. En effet, les instances civiles et ecclésiastiques refusèrent d'accorder au cours primaire supérieur la valeur d'un véritable cours secondaire donnant plein accès à l'université. Dans les années cinquante, des nouveaux leaders sociaux s'en prirent à la faiblesse de cet enseignement, et, dans leurs diatribes, dénigrèrent à la fois l'Église, le Département de l'Instruction publique, le système d'enseignement et les congrégations. Des religieux entrèrent dans la ronde des protestataires, dont le plus connu, mais non le seul, il s'en faut, fut le Frère Un Tel, pseudonyme de Jean-Paul Desbiens, mariste ${ }^{32}$.

La protestation des religieux fut avant tout active. Dès 1943, et surtout après.1950, les frères créèrent, en opposition aux instances gouvernementales et épiscopales, à l'exception de $\mathbf{M}^{\mathrm{gr}}$ Charbonneau, archevêque de Montréal, des classes de douzième et de treizième année permettant l'accès aux écoles ou facultés de sciences, de commerce, de génie ou à l'école polytechnique. Des programmes spéciaux ouvrirent la porte, après le secondaire, aux autres facultés universitaires, à l'exception du droit, de la médecine, de l'art dentaire et de la théologie, lieux de formation des défenseurs des humanités classiques. À Montréal, les écoles des frères alimentèrent l'Uńiversité McGill, anglo-

\footnotetext{
${ }^{30}$ AFFE, Projet de fondation d'un Institut des Sciences religieuses, 23 mars 1954, 1 p; AFFE, Procès-verbal, 4 septembre 1954, 1 p.; AFFE, Rapport de la souscommission des études, 1959, p. 1.

${ }^{31}$ AFFE, Procès-verbal, 4 septembre 1954, 1 p.; AFFE, Lettre de $\mathbf{M}^{\mathrm{gr}}$ CharlesOmer Garant au Père Louis-Joseph Lefebvre, CSV, 2 mars 1955.

${ }^{32}$ AFFE, Procès-verbal, 7 décembre 1957, p. 2; Georges Croteau, Les frères éducateurs, pp. 151-163, 188-204.
} 
phone et protestante, à la consternation des autorités diocésaines. Ces initiatives surgirent surtout en province, notamment dans la Mauricie, l'Estrie et le Saguenay Lac Saint-Jean et la région de Québec. Les frères pouvaient compter sur l'appui d'une part de la population et du clergé paroissial, des doyens préoccupés du développement de leur faculté, d'associations nationalistes telle la Société Saint-Jean-Baptiste ${ }^{33}$.

Le soutien des nationalistes s'explique par le fait que les frères faisaient valoir avec force, dans la défense de l'enseignement secondaire public, la préparation des francophones à un rôle de premier plan dans l'industrie, le commerce, les sciences, la technologie, les métiers de pointe. D'après eux, il importait au premier chef que les Canadiens français cessent d'être une main-d'oeuvre à bon marché vouée à des occupations serviles et qu'ils contrôlent le développement de leur pays. Cela exigeait de mettre fin au monopole des humanités classiques dans l'accès à l'université, de diversifier l'enseignement secondaire ou post-secondaire dans le respect de la diversité des intelligences, de permettre à chaque enfant de développer ses talents indépendamment de ses ressources financières ou de sa localisation géographique. C'était reprendre, à peu de choses près, les idées avancées dans les années vingt, à la différence que les programmes proposés étaient plus affinés par suite d'une formation intellectuelle plus poussée des frères, d'une pratique du secondaire déjà éprouvée et de l'apport de méthodes pédagogiques nouvelles.

Les mémoires eurent peu d'efficacité immédiate, aussi bien celui de la Fédération soumis à la Commission Tremblay en 1952 que celui retenu à l'intention de la Commission du Programme de l'Université Laval en 1958, sans oublier les écrits de particuliers ${ }^{34}$. Bien plutôt, les commissions scolaires prirent le contrôle exclusif de l'enseignement secondaire public sur lequel allaient empiéter les humanités avec l'introduction décisive, en 1954, de sections classiques dans les écoles publiques. Celles-ci apparaissaient, même à certains frères, une solution à l'impasse consécutive à la réduction obligatoire, en 1960, du cours

\footnotetext{
${ }^{33}$ Ibid., pp. 163-84. Le lecteur trouvera dans ces pages une description détaillée et située des interventions des frères dans l'enseignement secondaire public. Cette description n'est toutefois pas exhaustive, car l'auteur n'a retenu que le plus significatif. Soulignons la création de l'Université de Sherbrooke en prolongement des initiatives du Frère Théode, FSC, réussissant à déjouer les règlements scolaires officiels.

${ }^{34}$ Georges Croteau, Les frères éducateurs, pp. 204-12; AFFE, Mémoire sur la coordination de l'enseignement à ses divers degrés, 3 janvier 1952, p. 6; AFFE, Mémoire présenté à la Commission du Programme de la Faculté des Arts de Laval, Québec, 14 mars 1958, $15 \mathrm{p}$.
} 
secondaire à la onzième année et à l'allégement de son programme en mathématiques et en sciences. Le cours issu du primaire supérieur n'était habilité qu'à dispenser une formation de culture générale ne donnant plus accès à l'université, même dans les disciplines telles le génie, les sciences, le commerce. Non sans afficher un certain désarroi devant cette situation inédite, les enseignants religieux se centrèrent sur le renouvellement pédagogique et l'adaptation des programmes à la clientèle scolaire. Ils devaient toutefois limiter les corrections aux seuls établisscments locaux et s'en remettre à l'ébauche d'une réforme scolaire dont la formulation officielle leur échappait pour une large part ${ }^{35}$.

L'étau se resserrait de plus en plus sur les frères enseignants jouissant encore d'appuis solides dans les milieux où ils oeuvraient ou de la part d'agents sociaux défendant des intérêts propres. Cet appui avait permis des initiatives efficacement protestataires entérinées ou mises de l'avant par une Fédération très active grâce à un secrétaire, le Frère M.-Cyrille des Écoles chrétiennes. D'autres congrégations que les CSV ou les FEC s'affirmèrent dans l'enseignement secondaire public, principalement les FMS, les FIC, les FSC ou la CSC. Le discours congréganiste, en matière d'éducation, de recrutement et de prestige social, se fit plus directement revendicatif vu les réclamations des enseignants laïques et l'opposition durcie de constataires sociaux, des congrégations cléricales, des officiels civils ou ecclésiastiques. Ces derniers se proposèrent, à la fin, d'édifier une réforme scolaire dont ils auraient la direction exclusive tout en faisant appel à l'exécution des congrégations. Du même coup, la «base sociale" de celles-ci se révéla fragile en vertu d'une extension géographique sans augmentation proportionnelle des effectifs et de la centralisation plus poussée des pouvoirs scolaires. Cette perte structurelle allait être compensée, pour un temps, par le resserrement des forces congréganistes abandonnant une part de leurs particularités.

${ }^{35}$ Cf. note précédente. AFFE, Lettre du F. Elzéar au F. M.-Cyrille, FEC, 9 octobre 1959, 1 p.; Frère Un Tel, «M. Roland Vinette, poète surréaliste», Le Devoir, 23 avril 1960, p. 2; AFFE, Gustave Fortier, FEC, Genèse d'une décadence, 7 novembre 1963, 2 p.; AFFE, Commission des Frères des Écoles secondaires. Historique, s.d., $1 \mathrm{p}$. 


\section{LES FRÈRES ENSEIGNANTS, DES AGENTS DE TRANSITION AU SERVICE D'UNE RÉFORME SCOLAIRE BUREAUCRATIQUE (1960-1970).}

Au tournant des années soixante, les supérieurs provinciaux travaillèrent à une plus grande efficacité du secrétariat moyennant un personnel plus nombreux, l'assignation d'un délégué permanent des frères des écoles secondaires, de meilleurs outils de travail et la formulation de mandats précis aux comités ou commissions. Ceux-ci furent portés respectivement au nombre de sept et de six, auxquels s'ajoutaient les divisions de la Commission des Frères des Écoles secondaires ${ }^{36}$. La pratique du religieux éducateur était considéré dans toutes ses dimensions, de même que l'administration des congrégations et leurs relations avec d'autres agents sociaux. On s'empressa aussi de réaménager les locaux du secrétaire et de son adjoint, de l'économe et des responsables des comités ou commissions ${ }^{37}$, à l'image d'ailleurs des organismes civils.

Ces changements, effectifs déjà depuis la fin des années cinquante, furent consignés dans les règlements généraux publiés en 1962. Ils fixaient dans le détail des façons de faire remontant aux origines ou apparues dans la gestion du quotidien si ce n'est à la suite de faits menaçants. Les objectifs de la Fédération étaient définis comme suit: aider la cause des frères éducateurs au Canada, c'est-à-dire favoriser le développement de la vie religieuse et faire progresser l'éducation chrétienne de la jeunesse. Pour ce faire les Règlements recommandaient une plus grande coopération entre les congrégations, la «culture des vocations", l'attention aux courants sociaux, une information positive

\footnotetext{
${ }^{36} \mathrm{La}$ FFE comprenait sept comités: la vie religieuse, les vocations, l'apostolat, la vie professionnelle et sociale, les finances, la publicité, la direction de Jesus Pastor; six commissions: les directeurs d'études, les directeurs des scolasticats-écoles normales, les maisons de formation, les frères des écoles secondaires, les procureurs, l'information sur les frères éducateurs. La Commission des Frères des Écoles secondaires chapeautait des sous-commissions correspondant aux disciplines suivantes: la religion, la philosophie, les mathématiques, les sciences, le français, le commerce, l'anglais (AFFE, La Fédération des Frères Éducateurs du Canada, janvier 1961, 1 p.).

${ }^{37}$ AFFE, La Fédération des Frères Éducateurs du Canada, janvier 1961, 1 p.; AFFE, Procès-verbal, 25 septembre 1961, pp. 2-3; AFFE, Réorganisation des comités des provinciaux, octobre 1961,3 p; AFFE, F. M.-Cyrille, FEC, Réorganisation $d u$ secrétariat des Frères, 14 octobre 1961, 4 p.
} 
du public sur le rôle des frères en éducation ${ }^{38}$. Dans le but de signifier visiblement l'existence des frères, la Fédération transporta son siège social à Québec, au centre politique du pays, et un Bulletin à large diffusion vit le jour en $1967^{39}$.

En 1968, les supérieurs ne s'entendirent pas sur la redéfinition des objectifs de la Fédération et rééditèrent tels quels les Règlements de 1962. L'année suivante, la Fédération devint la Conférence des Frères provinciaux intégrée en 1970 à la Conférence religieuse canadienne (CRC) ${ }^{40}$. Des douze congrégations membres ${ }^{41}$ en 1969 , certaines avaient quitté les rangs de la Fédération, dont ses initiateurs, les CSV, alléguant qu'elle faisait double emploi avec la CRC ou qu'elle ne répondait plus aux besoins de l'heure. Depuis un moment déjà, un grand nombre de frères se désintéressaient des délibérations des supérieurs, les considéraint dépassées, inopportunes, coupées des problèmes réels des religieux actifs. Ceux-ci avaient revendiqué en vain, dès le début des années soixante, une plus grande participation aux discussions de la Fédération, mais les provinciaux s'étaient réservé l'exclusivité décisionnelle au nom du voeu d'obéissance et de la discrétion exigée par certaines questions ${ }^{42}$. La conférence des provinciaux s'évanouit au milieu de dissensions engendrées par les bouleversements de la réforme scolaire et le dernier concile.

L'évolution de la Fédération reflétait celle de l'insertion des frères dans le système d'enseignement. Nous allons retracer les points majeurs de cette évolution. En 1961, le gouvernement québécois accepta de

\footnotetext{
${ }^{38}$ AFFE, Règlements généraux, 1962, p. 2.

${ }^{39}$ AFFE, Procès-verbal de la réunion des membres de l'exécutif, 8 mai 1963, p. 1; AFFE, Lettre du F. Hector Deshaies, FEC, aux supérieurs provinciaux, 30 décembre 1964. Il s'agit du Bulletin des Frères Éducateurs dont les livraisons, à périodicité variable, s'étendent de 1967 à 1971, avec un tirage atteignant jusqu'à trois mille exemplaires.

${ }^{40}$ AFFE, Procès-verbal, 1er mars 1968, p. 3; ler mai 1968, p. 2; 9 novembre 1969, 4 p.; AFFE, Henri Gingras, FIC, Aux quelques 5000 confrères de la Fédération des Frères Éducateurs, 1969, 1 p.

${ }^{41}$ Les frères éducateurs étaient au nombre de 6103 en 1962, de 5467 en 1967 et de 4 095, en 1971 (CRC, Statistiques, 1979, pp. 22-6). En outre, la Fédération compta jusqu'à 13 congrégations, soit en 1966. D'autres congrégations s'étaient ajoutées: les Religieux de Saint-Vincent de Paul, les Frères de Notre-Dame de Lourdes et les Frères de Saint-Louis de Gonzague. Ces deux dernières congrégations furent remplacées en 1969 par l'Ordre Hospitalier de Saint-Jean de Dieu.

42 AFFE, Procès-verbal de la réunion de l'exécutif de la Fédération, 29 janvier 1965, p. 1; AFFE, Procès-verbal d'une réunion informelle, ler novembre 1968, p. 1; ler mars 1968, p. 3.
} 
financer la construction d'écoles normales congréganistes à la condition qu'elles se regroupent. Il fut fait ainsi à Québec et à Montréal, et deux seules congrégations conservèrent leur scolasticat propre: les Frères de l'Instruction chrétienne à Laprairie et les Clercs de Saint-Viateur à Rigaud. De part et d'autre des immobilisations importantes furent commandées par la formation prolongée des nouvelles recrues ou par l'obligation faite aux enseignants d'obtenir des diplômes supérieurs en pédagogie. Les édifices inaugurés autour de 1965 furent transformés en collèges d'enseignement général et professionnel dès 1968, car l'enseignement pédagogique relevait désormais des universités, notamment de l'Université du Québec. La clientèle de ces maisons devint rapidement à majorité laïque et mixte vu les nécessités financières et le tarissement du recrutement. Dans ces conditions, les congrégations purent maintenir, après l'achèvement de la réforme scolaire, la direction et le professorat dans l'enseignement secondaire supérieur privé ${ }^{43}$.

En 1959, les supérieurs provinciaux décidèrent, à la demande expresse de leurs sujets, de donner un nouvel élan à la Commission des Frères des Écoles secondaires. Des religieux reconnus pour leur compétence travaillèrent dans des sous-commissions au renouvellement des programmes scolaires en accord avec les orientations du Ministère de la Jeunesse et les besoins du milieu éducatif ou des régions. Regroupant des frères de toutes congrégations et de tous les coins du pays, ces sous-commissions produisirent des guides pédagogiques, rédigèrent des plans de cours, firent des sessions à l'intention des enseignants tant laïques que religieux. Elles constituèrent un lieu de création au service des établissements scolaires et des instances civiles en charge de l'enseignement. Ce fut un véritable laboratoire auquel s'associèrent les laïcs dès 1960, et de plus en plus par la suite. Ceux-ci prirent la direction des commissions avec la syndicalisation des enseignants congréganistes en $1965^{44}$. À partir de cette date, le Ministère de

\footnotetext{
${ }^{43} \mathrm{AFFE}$, Rapport de la réunion des provinciaux et de la commission des Écoles Normales, 29 janvier 1967, 1 p.; AFFE, Les scolasticats-écoles normales. Historique, s.d., 1 p.; Le Collège de Rigaud ferma ses portes en 1970, mettant fin au rêve d'un CEGEP viatorien (Paul-André Turcotte, L'éclatement d'un monde, p. 264). Le scolasticat de Laprairie fut intégré à un ensemble éducatif de la rive sud. Les Collèges MarieVictorin de Montréal et Notre-Dame de Foy de Cap-Rouge continuent encore aujourd'hui de dispenser l'enseignement collégial ou secondaire supérieur.

${ }^{44}$ L'adhésion des religieux à la Corporation des Enseignants Catholiques du Québec fut décidée le 22 février 1965 (AFFE, Procès-verbal, 22 février 1965, 1 p.), et celle à l'Alliance des professeurs de Montréal, en juillet 1966 (AFFE, Entente entre les dirigeants de l'Alliance et les représentants des communautés religieuses enseignantes, juillet 1966, 5 p.).
} 
l'Éducation et les universités s'adressèrent aux supérieurs pour obtenir des professeurs aptes à l'enseignement supérieur ou des fonctionnaires capables de mener à terme la réforme scolaire, notamment en travaillant à la direction générale des programmes et des examens ${ }^{45}$. La remise de l'éducation à l'État par l'Église avait signifié, pour les frères, la possibilité de pouvoir concrétiser les virtualités de leur projet éducatif refoulé pendant des décennies par le Comité catholique, les clercs des collèges classiques et leurs alliés.

Le travail des commissions pédagogiques se continua jusqu'en 1968. À cette date, la Corporation des Enseignants catholiques du Québec (CEQ) décida de les dissoudre, avançant que les particularités régionales ne pouvaient s'accorder avec la centralisation décisionnelle du syndicat. Les enseignants de quelconque discipline gardaient toutefois le droit de se regrouper en association. Cette disposition correspondit à la déconfessionnalisation de la CEQ et à son orientation prioritaire vers la défense des intérêts socio-économiques de ses adhérents.

Cette nouvelle orientation déconcerta un grand nombre de religieux. $\mathrm{Ne}$ les avait-on pas assurés, au moment de leur adhésion, que la CEQ ne prendrait pas le caractère d'un syndicat ouvrier et qu'elle se centrerait sur la compétence du corps professoral, les services à la population et l'éthique professionnelle en vue de hausser le prestige social de l'enseignant, ce qui supposait une rémunération et des conditions de travail adéquates? Sur ces promesses et sur les recommandations de clercs versés dans les sciences sociales, les congrégations avaient accepté la syndicalisation. Elles avaient espéré recueillir de leur adhésion massive le plein droit de parole auprès des instances civiles qui entendaient désormais communiquer exclusivement avec le corps enseignant le plus représentatif. Elles avaient insisté pour qu'on leur reconnaisse les particularités suivantes: le travail en équipe communautaire, la dispensation de services appréciés par la population, l'acceptation de tâches non rémunérées, le droit à la dissidence si le bien des enfants n'était pas respecté, l'abstention à des activités sociales inconciliables avec l'état de vie religieux. Ces clauses furent progressivement ignorées, et les

\footnotetext{
${ }^{45}$ AFFE, Procès-verbal de la réunion de la Commission des Frères des Écoles secondaires, 15 mai 1960, p. 1; AFFE, Léandre Dugal, CSV, „Éditorial ", Service (janvier 1965) pp. 1-2; AFFE, Procès-verbal, 6 mars 1965, p. 2; AFFE, Lettre de M. Jean-Marie Joly au F. Louis-Bertrand, FSG, 27 janvier 1965. Nous nous appuyons aussi sur les témoignages des Frères Léandre Dugal, CSV, et Roger Lemay, FEC.
} 
enseignants congréganistes se trouvèrent obligés de se soumettre à des directives dictées par une bureaucratie indifférente ou opposée, d'après nombre d'entre eux, à leur conception de l'éducation ${ }^{46}$.

$\mathrm{Au}$ moment même de l'apogée des sous-commissions de l'enseignement secondaire, des évêques et l'Office cathéchistique provincial incitèrent les frères à se centrer sur le domaine strictement religieux à l'école. La pratique dominicale était à la baisse, surtout à Montréal, et l'Église affrontait une chute de crédibilité sociale. L'enseignement de la cathéchèse conçu aussi bien comme témoignage personnel que comme transmission de connaissances renouvelées apparut un moyen de freiner ce mouvement, notamment au sein de l'école particulièrement touchée par les remous de la révolution tranquille. Se soumettant à cette politique, les frères s'inscrivirent, en nombre croissant, à des cours de catéchèse, de sciences religieuses ou de théologie. Certains tentèrent de s'adonner à l'enseignement de la religion à temps plein et non à temps partiel comme depuis toujours. Les difficultés de cet enseignement dans les écoles régionales polyvalentes en déroutèrent plus d'un. À la fin des années soixante, on était loin des $40 \%$ de frères cathéchètes souhaités en 1961 par les protagonistes du projet.

Une autre composante de la politique ecclésiastique était la formation des maîtres chrétiens dans les écoles normales masculines dirigées par les congrégations. Cela justifia l'ouverture des scolasticats à des futurs enseignants laïques désireux d'y trouver une formation intellectuelle, pédagogique et chrétienne de niveau élevé. L'orientation des normaliens vers les universités mit fin à la formation commune des religieux et des laïcs dans les établissements congréganistes. Cette formation commune avait été préconisée dans le but de constituer une cohorte d'élite se portant à la défense de l'éducation chrétienne

\footnotetext{
${ }^{46}$ AFFE, Procès-verbal, 5 juin 1964, p. 2; AFFE, F. Hector Deshaies, FEC, "Adhésion des éducateurs religieux à la corporation des instituteurs catholiques", La Profession (novembre 1964), p. 2; AFFE, Compte rendu de la réunion de la CIC avec le FFE et l'AREQ, 13 juin 1966, 6 p.; AFFE, Lettre de M. Léo Garant, président de la CIC, au F. Hector Deshaies, secrétaire-adjoint de la FFE, 1964; AFFE, Mémoire, 1964, pp. 2-10; AFFE, F. Laurier Labonté, FIC, Vie religieuse et affiliation syndicale, 1964, pp. 3-10.
} 
et de la confessionnalité scolaire dans une société rompant avec le catholicisme traditionnel ${ }^{47}$.

Non seulement la politique d'assurer un caractère effectivement chrétien à l'école publique ne s'imposa pas, mais bien plus encore, les frères furent intégrés indistinctement dans le système public d'enseignement et les syndicats. Une bureaucratie bicéphale canalisait désormais les forces congréganistes vers l'application de la réforme scolaire. Dans la fouléc d'un renversement perceptible dès la seconde moitié des années cinquante, les congrégations dirigèrent un nombre significatif de leurs membres vers les missions lointaines. Ainsi 345 frères partirent oeuvrer en pays étrangers, de 1954 à 1958, et le nombre des frères missionnaires, de 542 qu'il était en 1960, atteignit 685 en 1966. Ce nombre se maintint dans la suite.

Dans le même mouvement, le Frère Jean-Paul Desbiens et le sousministre de l'Éducation, Arthur Tremblay, mirent sur pied, en 1967, le projet MIRE, c'est-à-dire le Ministère de l'Éducation pour Religieux, dont l'objectif était d'accorder à des régions défavorisées ou éloignées une équipe de frères, d'au minimum trois, chargés de créer des services éducatifs adéquats ou de consolider ceux existants. Ces régions pourraient de la sorte bénéficier de compétences dans des domaines où les ressources locales étaient insuffisantes si ce n'est inexistantes. Des équipes intercongréganistes furent créées à cet effet, mais il ne semble pas que l'opération eut l'envergure souhaitée ${ }^{48}$. Elle ne constitua pas moins, avec les missions lointaines et d'autres initiatives, la tentative de créer des lieux où les frères pourraient poursuivre une action originale.

\footnotetext{
${ }^{47}$ AFFE, Projet d'un Institut de Pastorale, 3-5 janvier 1961, 3 p.; AFFE, Mémoire Rigaud, 17 juin 1961, 3 p.; AFFE, Norbert Fournier, CSV, La formation doctrinale des frères éducateurs en sciences religieuses, septembre 1961, 3 p.; AFFE, F. M-Cyrille, FEC, Petit mémoire du secrétariat en vue de l'orientation à longue portée des projets de notre fédération, 18 septembre 1961, 3 p.; AFFE, F. Yvon Poitras, FIC, "L'éducation chrétienne aujourd'hui", Frères éducateurs, Cahier I, 1966-1967, s.p.; AFFE, Procès-verbal, 5 juin 1964, p. 2; AFFE, Lettre du F. Louis-Bertrand, FSG, à M. Léo Garant, 20 juin 1963.

${ }^{48}$ AFFE, Renseignements divers, 30 décembre 1959, p. 1; AFFE, F. M-Cyrille, FEC, "25, 30, 60 ans...», p. 38; AFFE, Mémoire de la Fédération des Frères Éducateurs à la Commission Royale d'Enquête sur l'Éducation, mai 1962, p. 2; AFFE, Statistiques, 1966; AFFE, MIRE, 26 avril 1967, 8 p.
} 
Le discours relatif à l'éducation suivit de près l'insertion des enseignants religieux dans le système d'enseignement. À la Commission Parent, la Fédération exprima le souhait, en 1962, que l'école secondaire publique réponde davantage aux besoins de la population locale et que ses programmes, accessibles à tous les élèves doués, conduisent à l'université. C'était démocratiser l'école et devoir rompre avec un enseignement d'inspiration française caractérisé par l'apprentissage des langues anciennes, la sélection élevée de la clientèle, l'accession confinée à une minorité aisée, la préparation d'une élite sociale. Une réforme s'imposait donc, que les congrégations n'avaient cessé de proposer depuis les années vingt. Cette réforme était conciliable, ajoutait-on, avec un enseignement privé restreint, autre que celui des collèges classiques et se distinguant par des objectifs éducatifs élevés. L'année suivante, la Fédération des Frères éducateurs et l'Association des Religieuses enseignantes du Québec (AREQ) précisèrent qu'il revenait à l'État de coordonner les initiatives privées en matière pédagogique et non d'en dicter les principes. En effet, les religieux et religieuses mettaient de l'avant le devoir de faire valoir au bénéfice de la population entière leur expérience éducative séculaire ${ }^{49}$.

Par la suite, les congrégations actives dans le secteur public se réjouirent de ce que le Rapport Parent endossait largement leurs vues sur l'éducation, notamment sur l'humanisme scientifique, la pédagogie active, la méthode expérimentale, mais elles n'étaient pas sans craindre une mainmise de l'État. C'est ce que signifia l'achèvement de la réforme scolaire établissant un enseignement néolibéral axé sur la transmission des connaissances et la préparation au marché du travail dans de vastes complexes scolaires. Des religieux de diverses congrégations et la Fédération opposèrent une école d'inspiration chrétienne, à distinguer de la confessionnalité juridique, qui soit une école enracinée dans le milieu, forte d'un corps professoral à haute compétence pédagogique, capable de formation intellectuelle, humaine et chrétienne de la jeunesse dans des établissements à taille réduite et aménagés en sections correspondant aux étapes de la formation intellectuelle et à la psychopédagogie de la clientèle scolaire. Mais en même temps, les promoteurs du projet reconnaissaient la difficulté de l'appliquer dans des écoles regorgeant

\footnotetext{
49 AFFE, Mémoire de la Fédération des Frères enseignants à la Commission Royale d'Enquête sur l'Éducation, mai 1962, pp. 4-17, 30-31; AFFE, Mémoire de la Fédération des Frères éducateurs du Canada et de l'Association des Religieuses enseignantes du Québec au Ministre de la Jeunesse, 26 mars 1963, pp. 1-2; AFFE, Procès-verbal des membres de l'exécutif, 8 mai 1963, p. 2; AFFE, Jean-Claude Ruest, FIC, «Editorial: science et humanisme», Service, (27 mars 1965), pp. 1-2.
} 
de «donneurs de cours». Les religieux n'avaient su, enchaînaient-ils, engendrer des fils spirituels prenant la relève ${ }^{50}$.

À la fin des années soixante, les frères se distribuaient en factions opposées: certains adoptaient les visées et les normes du Ministère de l'Éducation ou des syndicats; d'autres opposaient à ces derniers le projet éducatif rappelé par les organismes congréganistes; plus d'un s'en remettait à l'Église officielle et oeuvrait dans le réduit confessionnel de la catéchèse ou de la pastorale; d'aucuns quittèrent l'école publique de la réforme scolaire, gonflant le nombre des missionnaires, des enseignants des collèges privés, des préposés aux services congrégationnels internes, des initiateurs de projets nouveaux, si ce n'est le camp des reclus. En concomitance à une violente crise d'identité sociale, le projet d'action des congrégations enseignantes passa du singulier au pluriel.

Les frères se trouvaient finalement fortement minorisés, évacués dans une certaine mesure, par une réforme scolaire dont ils avaient été les inspirateurs, au moins indirects, et les exécutants. L'Église s'était retirée du champ de l'éducation, la centralisation étatique ou syndicale imposait son monopole, et le soutien aux congrégations était devenu particularisé et sans poids réel. Même la Fédération des Frères éducateurs s'était évanouie après avoir permis aux congrégations de faire valoir leurs vues auprès des instances éducatives grâce à une coordination interne imitant celle des concurrents. Toutefois cette coordination avait sécrété un écart sans cesse grandissant entre les supérieurs tournés vers les bureaucraties civiles ou ecclésiastiques et les subordonnés aux prises avec une pratique conflictuelle mettant en lumière la désuétude de certaines façons de faire.

$\mathrm{Au}$ même moment, les troupes entreprirent les chemins de la dispersion. Devant ce fait inédit, les congrégations cherchèrent à articuler leur "charisme propre» à la pluralité des projets d'action ou au réduit des besoins ecclésiastiques circonscrits au strictement religieux. Elles tentèrent d'échapper à une dépendance totale et de résoudre la crise d'identité de leurs membres. Des voies s'ouvraient, que d'aucunes empruntèrent: le repli sur elles-mêmes, le retrait vers d'autres lieux d'action, l'éparpillement des effectifs, à moins qu'un gonflement de la superstructure ne vînt confronter une base de crédibilité déticiente, pro-

${ }^{50}$ AFFE, Rapport Parent. Étude des structures des cours élémentaire et secondaire, 8-10 janvier 1965, 6 p.; AFFE, Claude Côté, FEC, "Nos responsabilités pastorales dans les écoles régionales», Frères éducateurs, Cahier III, s.p.; AFFE, Marcel Vézina, FEC, 1967: léthargie ou réveil?, avril-mai 1967, p. 5. 
mettant ainsi d'échapper à un renfermement total ou à une complète dissolution ${ }^{51}$. Les congrégations étaient à la quête d'une "base sociale» après être passées du statut de force sociale déterminante à celui de force sociale déterminée, après être entrées dans le processus de la pluralisation ramenant, suggérons-nous, la négociation des conflits à des dimensions d'abord internes.

\section{CONCLUSION}

L'action instituante des congrégations masculines dans l'enseignement public québécois, particulièrement au degré secondaire, se révèle connotée à un ensemble de facteurs: l'autonomie d'intervention en éducation, le soutien recueilli tant dans le milieu d'évolution qu'auprès d'agents sociaux intéressés, la concertation intercongréganiste, l'institutionnalisation de la Fédération des Frères Éducateurs, la subordination institutionnelle, civile ou ecclésiastique. D'autres questions paraissent en raccourci ou en filigrane: ce sont les conflits des congrégations avec d'autres agents sociaux, les contraintes économiques dans la pratique du projet protestataire, les sources d'identification sociale et les rapports entre langue, religion, nationalisme et classes sociales. Des questions dépassant les limites d'un article dont le propos a été d'esquisser un tableau, forcément à grands traits, de l'action des congrégations masculines dans le secteur public de l'enseignement québécois.

\section{Paul-André TURCOTTE \\ Université Saint-Paul, Ottawa.}

\footnotetext{
${ }^{51}$ C'est ce que nous avons montré dans notre ouvrage L'éclatement d'un monde, aux pages 263 à 289.
} 